\title{
Strategy and cost in investigating solitary pulmonary nodules
}

\author{
Michael K Gould, Glen A Lillington \\ Department of Medicine, Stanford University, 300 Pasteur Drive, Stanford, \\ California 94305, USA
}

Introductory articles

Likelihood of malignancy in a solitary pulmonary nodule: comparison of Bayesian analysis and results of FDG-PET scan

NA Dewan, CJ Shehan, SD Reeb, LS Gobar, WJ Scott, K Ryschon

Objective. To compare the probability of cancer in a solitary pulmonary nodule using standard criteria with Bayesian analysis and result of 2-[F-18] fluoro-2-deoxy-D-glucose-positron emission tomographic (FDG-PET) scan. Setting. A university hospital and a teaching Veteran Affairs Medical Center. Methods. Retrospective analysis of 52 patients who had undergone both CT scan of the chest and a FDG-PET scan for evaluation of a solitary pulmonary nodule. FDG-PET scan was classified as abnormal or normal. Utilizing Bayesian analysis, the probability of cancer using "standard criteria" available in the literature, based on patient's age, history of previous malignancy, smoking history, size and edge of nodule, and presence or absence of calcification were calculated and compared to the probability of cancer based on an abnormal or normal FDG-PET scan. Histologic study of the nodules was the gold standard. Results. The likelihood ratios for malignancy in a solitary pulmonary nodule with an abnormal FDG-PET scan was 7.11 (95\% confidence interval [CI], 6.36 to 7.96), suggesting a high probability for malignancy, and 0.06 ( $95 \% \mathrm{Cl}, 0.05$ to 0.07 ) when the PET scan was normal, suggesting a high probability for benign nodule. FDG-PET scan as a single test alone was more accurate than the standard criteria and standard criteria plus PET scan in correctly classifying nodules as malignant or benign. Conclusion. FDG-PET scan as a single test was a better predictor of malignancy in solitary pulmonary nodules than the standard criteria using Bayesian analysis. FDG-PET scan can be a useful adjunct test in the evaluaiton of solitary pulmonary nodules. (Chest 1997;112:416-22)

The cost of diagnosis: a comparison of four different strategies in the workup of solitary radiographic lung lesions

B Goldberg-Kahn, JC Healy, JW Bishop

Introduction. Due to the large numbers of negative results of sputum examinations in the face of malignant disease, we used a decision analytic model to determine whether, and under what conditions, sputum cytology (Spt) might have a cost-effective role to play in the approach to lung lesions. Methods. We constructed a decision analytic model to compare the utility of Spt, image-directed fine-needle aspiration (FNA), bronchoscopic examination (Bronch), and open biopsy (OBX) in the evaluation of lung lesions. Prevalence and cost data were derived from local databases and diagnosis-related groups. Diagnostic sensitivity (sens) and specificity (spec) of the tests were derived from the literature and local data. Output of the model was lowest cost per correct malignant diagnosis and included surgical treatment costs. We did not attempt to model survival data or morbidity. Sensitivity analyses were performed using cost, test sensitivity, and lesion size variables. Results. In the baseline case, a patient who is a surgical candidate with lesion size $2.8 \mathrm{~cm}$, prevalence of malignancy $=0.67$, FNA sens $=0.95$, Bronch sens $=0.80$, Spt sens $=$ $0.51, O B x$ is the best initial procedure with a cost per correct diagnosis of $\$ 12,888$. Sputum examination has the highest cost per correct diagnosis of $\$ 63,424$. FNA and bronchoscopy have cost per correct diagnosis of $\$ 21,543$ and $\$ 16,615$, respectively. Sens analysis shows that OBx is the preferred strategy in the workup of lung lesions in patients who are surgical candidates older than 30 years of age. Spt is the preferred 
strategy only when the patient is not a surgical candidate, the lesion size is large $(>4.7 \mathrm{~cm})$, and only if sputum sens is $>0.45$. Conclusions. Spt is not cost-effective in clinical practice, except when the patient has a large clinically unresectable lesion and the laboratory can demonstrate sufficient test sens. (Chest 1997; 111:870-6)

Medicine is a science of uncertainty and an art of probability

Sir William Osler

Management of the solitary pulmonary nodule (SPN) is often challenging. ${ }^{1}$ Since SPNs are not typically associated with symptoms, most are incidental discoveries. As the overall prevalence of malignancy in SPNs is high at $20-40 \%$, the primary goal of management is to identify and resect malignant nodules promptly. An important secondary goal is to avoid resecting benign nodules. The standard dictum since the 1950 s has been to resect all nodules promptly unless their benign nature could be established conclusively, assuming the subject is suitable for surgery in other respects. The accepted criteria for benignity included: (a) the presence of certain patterns of intranodular calcification, and/or (b) stability (no growth of the nodule for a period of two years or longer). More recently there has been a search for approaches which will decrease the incidence of unnecessary surgical resection of benign nodules and decrease the overall cost of management.

Estimating the probability of malignancy

It has long been accepted that an accurate estimation of the probability that an SPN is malignant would provide significant assistance in management decisions. Clinicians often attempt to estimate such probabilities intuitively, but a number of biases may confound this process. $^{2}$

Several groups of investigators have developed formal guidelines for estimating the probability of cancer (pCA) in SPNs. ${ }^{3-6}$ Predictions are based on patient characteristics and the results of non-invasive imaging studies, particularly computed tomographic (CT) scanning. These "predictor variables" include important patient characteristics such as "baseline" prevalence of malignancy in solitary nodules in the population under consideration, age, smoking status, and a prior history of extrapulmonary malignancy. Imaging criteria may of extrapulmonary malignancy. Imaging criteria may
include nodule location, diameter size, edge characterinclude nodule location, diameter size, edge characteristics, growth rate, calcification pattern on the CT
and cavity wall thickness if cavitation is present.

and cavity wall thickness if cavitation is present.
Likelihood ratios for each of the clinical or radiographic characteristics chosen may be combined using Bayes' theorem to update the probability of malignancy derived from the baseline prevalence. After calculating the pCA, decisions to take biopsy specimens, perform further imaging studies, carry out a diagnostic/therapeutic thoracotomy, or manage the patient expectantly can be taken accordingly, as will be discussed later. The revised probability estimate cannot differentiate between malignancy or benignity with certainty, but rather serves as a guide to subsequent decision making.

The validity of the use of likelihood ratios in Bayesian probability assessment depends upon certain assumptions that may or may not be valid. The two major ones are that the predictor variables are conditionally independent and that the baseline prevalence of malignancy employed in the calculations is appropriate for the population being studied. External validation of probability calculations with likelihood ratios derived from published data would require a very large study group, and this has not been done. However, in the study by Swensen et $a l^{6}$ an internal validation was achieved by developing the probability model with achieved by developing the probability model with
multivariate logistic regression in a large cohort $(n=$ $419)$ and testing it on a separate cohort $(n=210)$. The correlation was excellent.

Positron emission tomography (PET): first introductory article

The first introductory article by Dewan et al reports a retrospective evaluation of the diagnostic role of PET in 52 subjects with SPNs ${ }^{7}$ In these subjects PET was in 52 subjects with SPNs. In these subjects PET was
$95 \%$ sensitive, $87 \%$ specific, and $92 \%$ accurate for $95 \%$ sensitive, $87 \%$ specific, and $92 \%$ accurate for
detecting cancer. Other investigators have reported similar results for PET test performance, although large prospective studies have not yet been performed. ${ }^{8-10}$ The corresponding likelihood ratios for malignancy were 7.11 for a positive PET result and 0.06 for a negative PET study. Thus, the positive predictive value and negative predictive value of PET were both high. The likelihood ratio of 7.11 for a positive PET study was greater than the likelihood ratios for each one of the "conventional" predictor variables employed by Cummings et $a l,{ }^{4}$ and all but one (cavity wall thickness) of the characteristics identified by Gurney et al. ${ }^{5}$

Using a baseline prevalence of malignancy in SPNs of $40 \%$, Dewan et al calculated that a likelihood ratio of 7.11 for a positive PET indicated a pCA of $82.4 \%$, and a likelihood ratio of 0.06 for a negative PET indicated a pCA of $3.8 \%$. When they compared these probability estimates with those obtained using the standard clinical and CT criteria, they found that the PET based estimates were more accurate for predicting (or excluding) cancer in individual subjects. This is not surprising since be particularly sensitive or specific. More surprising was their finding that the PET based probability estimates were more accurate than estimates obtained by combining the PET results with the standard clinical and CT criteria. In other words, the addition of clinical and CT characteristics to the calculation had a negative impact on diagnostic accuracy - that is, one would do better to igr would do better to ignore the standard criteria and base strategies for subsequent management solely on the sults of PET

This seemingly counter-intuitive result is not easily explained. It seems that some of the likelihood ratios underlying the standard criteria are not readily applied generally, at least with respect to this particular study sample. The 52 subjects studied by Dewan et al almost certainly differed from the populations used to develop the likelihood ratios with respect to both observed and unobserved characteristics. One notable observed difference was the extremely high overall prevalence of malignancy $(71 \%)$ in the population studied by Dewan et al. Since the prevalence of malignant SPNs was much lower in most of the studies used to develop the likelihood ratios for the standard criteria, it is not surprising that the latter would underestimate the pCA 
in many of the cases studied by Dewan. This problem in generalising is not unique to this example. Whenever clinical trial results are used to support decision making in individual patients or other groups of patients, the applicability of the findings of the trial (external validity) must be considered.

It is axiomatic that spectrum bias in the initial studies of a new test will give somewhat inflated values for sensitivity and specificity. Nevertheless, it seems very likely that PET will prove to be very useful in the diagnosis and management of SPN and may eventually replace needle aspiration biopsy in many situations. If the results of these preliminary studies are confirmed, the negative predictive value of PET will be high and expectant (non-surgical) management in many or most patients who have a negative PET will be a reasonable patients who have a negative PET will be a reasonable
choice. If PET imaging substantially reduces the number choice. If PET imaging substantially reduces the number of unnecessary surgical resections of benign nodules, it will also probably prove to be highly cost effective. A large multicentre Veterans Administration Cooperative Study is currently underway to define better PET performance and cost effectiveness for SPN diagnosis and for tumour staging.

Furthermore, the high sensitivity of PET scanning may encourage local but potentially curative resection of SPNs using video assisted thoracoscopy whenever thoracotomy and lobectomy are considered inappropriate because of co-existing morbidity.

DIAGNOSIS OF SPN USING DECISION ANALYSIS

Prior to the availability of PET, three formal decision analysis studies were published on the effectiveness of various strategies for SPN management. ${ }^{311} 12$ While the design of the decision trees and the choice of probability variables differed somewhat, the conclusions were similar. The average utility (expected value) of each strategy was quantified in terms of average years of expected survival. The level of pCA had the greatest influence survival. The level

As might be expected, the strategy of immediate thoracotomy was optimal when the pCA was relatively high. The strategy of watchful waiting, which included prospective monitoring with serial chest radiographs followed by prompt resection if growth occurred, was (o other strategies by a small margin when pCA was low, usually $<10 \%$. The study by Cummings et al included a mechanism for quantifying the "hazard of delay" inherent in such watchful waiting strategies. ${ }^{11}$ Over relatively wide intermediate ranges of values for $\mathrm{pCA}$, the strategy of needle aspiration biopsy had a marginally greater utility than immediate thoracotomy and a more obvious advantage over the watchful waiting strategy.

It has long been recognised that factors other than the duration of life should be considered in quantifying the effectiveness of a strategy. For example, five years of life in chronic pain or disability is less desirable than five years of survival without symptoms. Adjustments for long term reductions in the quality of life were not employed in the above-mentioned studies, nor were there any formal conside adjustments will typically reduce calculated effectiveness in strategies (such as thoracotomy) that produce chronic complications in some instances.

INTERPRETING THE RESULTS OF COST EFFECTIVENESS

STUDIES

In cost effectiveness analysis health effects are measured in natural units such as life years saved or cases of pneumonia prevented. Analysis of cost effectiveness always compares two or more interventions and incremental cost effectiveness ratios are calculated by dividing the difference in costs by the difference in health effects between competing interventions. A special type ures health effects in terms of both quality and duration of life. Such an approach is sometimes called a cost utility analysis. The "quality adjusted life year" (QALY) is the preferred denominator for this type of analysis, although even this measure is less than perfect. ${ }^{1314}$ However, using QALYs as the measure of effectiveness produces the most generalisable results so that diferent health care inwithin and across different diseases. For example, in acute myocardial infarction, if the incremental cost effectiveness of tissue plasminogen activator compared with streptokinase is $\$ 30300$ per additional QALY saved, this value should be judged in the context of the calculated cost effectiveness of other commonly accepted health care interventions that serve as benchmarks. Occasionally, cost tres effectiveness studies report average cost effectiveness ratios in which the costs of a single intervention are divided by its health effects. Average cost effectiveness ratios are difficult to interpret and potentially misleading since they do not reveal the incremental benefits and costs of adopting an intervention compared with some alternative.

Many other considerations arise when interpreting Many other considerations arise when interpreting
the results of cost effectiveness studies: was the analysis performed from a societal perspective or some other perspective; how did the authors determine costs; how far into the future were costs tallied; how were costs and health effects discounted? Answers to these questions may have a major impact on the validity and general applicability of the results of such studies.

When an intervention or strategy is found to be less expensive and more effective in a cost effectiveness study it is often called a dominant alternative. If the study results are valid and applicable to the reader's setting, the strategy should be adopted. More commonly, the more effective strategy is also more expensive. In such cases it is not obvious whether the improvements in health justify the increased costs. Thus, cost effectabout competing health care interventions but do not
obviate the need for value judgments in many instances.

\section{Cost effectiveness in lung cancer staging and} diagnosis: second introductory article

In 1988 Black and associates published a well designed cost effectiveness study on a special subset of SPNs- the T1N0M0 pulmonary nodule-concentrating on the value of routine mediastinal CT studies..$^{15}$ As many of the assumptions employed may no longer be valid, their conclusion that CT scanning is cost effective may need to be re-examined and revised. The value of sputum cytological studies, a traditional technique for lung cancer diagnosis, has recently become a further focus of attention. Two groups of researchers have reached opposite conclusions about the cost effectiveness of this
diagnostic test.

In the second introductory article Goldberg-Kahn et $a l^{16}$ reported decision analysis to compare the cost in the USA per correct diagnosis for various combinations of sputum cytology, fine needle aspiration biopsy, bronchoscopy, and open biopsy. In their baseline analysis they concluded that proceeding directly to open biopsy 
(thoracotomy) resulted in the lowest average cost effectiveness (\$12 888 per correct diagnosis). Conversely, a strategy which included sputum cytology as the initial test (followed by bronchoscopy, needle biopsy and/or open biopsy) resulted in the highest cost (\$63 424) per correct diagnosis.

This result seems counter-intuitive, especially since the total cost for all four procedures was only $\$ 14090$. However, if the number of correct diagnoses was much lower for strategies involving initial sputum cytology than for open biopsy as the initial test, then the cost per correct diagnosis for initial sputum cytology could conceivably be much higher than the total cost of all four procedures. The authors did not report the number of correct and incorrect diagnoses for each strategy, but of correct and incorrect diagnoses for each strategy, but the large difference in cost per correct diagnosis could
only be explained if the percentage of incorrect diagnoses only be explained if the percentage of incorrect diagnoses obtained with the initial sputum cytology strategy was very large. This may have been so, but it is disingenuous to argue that the investigatory strategy was a failure because the initial use of sputum cytology gave falsely negative results when subsequent tests within the strategy were positive.These considerations aside, two fundamental problems limit the validity and applicability of the results of this analysis. Firstly, and most important, the authors reported average cost effectiveness ratios rather than incremental cost effectiveness ratios. As previously mentioned, this produces results that are difficult to interpret and potentially misleading. Secto interpret cost per correct diagnosis as a measure of cost effectiveness since no easonable benchmarks exist for comparison.

A decision analytical approach to evaluate the cost and effectiveness of strategies that included sputum cytology compared with those that did not was also used by Raab et al. ${ }^{17}$ These authors expressed their results in terms of incremental costs and life expectancies, so their results are more easily interpreted and generalised. Like Goldberg-Kahn et al, they evaluated various combinations of sputum cytology, fine needle aspiration biopsy, bronchoscopy, and open biopsy (via thoracoscopy). In addition, they included the possibility of expectant management for those with nondiagnostic results on bronchoscopy and needle biopsy (with or without non-diagnostic initial sputum cytology). Furthermout this group considered centrat and peripheral lesions separately, and assumed that the test peripheral lesions separately, and assumed that the tes
performance for sputum cytology, bronchoscopy, and performance for sputum cytology, bronchoscopy, and
needle biopsy varied depending on whether a lung lesion needle biopsy varied depending on whether a lung lesion of this model is that the authors incorporated estimates of morbidity and mortality for each of the diagnostic procedures.

They found that, for central lung lesions, calculated life expectancies were similar for strategies that used initial sputum cytology and those strategies in which sputum cytology was not employed. One exception was that performing sputum cytology prior to open biopsy via thoracoscopy increased life expectancy by an average of 107 days compared with using open biopsy as the initial test. With respect to costs, sputum cytology as the initial test was always less expensive than a comparable strategy without initial sputum cytology. Thus, in contrast to the findings of Goldberg-Kahn et al, strategies trast to the findings of Goldberg-Kahn et al, strategies
which included sputum cytology almost always inwhich included sputum cytology almost always in-
creased life expectancy and always resulted in lower costs. The optimal diagnostic strategy consisted of sputum cytology followed by bronchoscopy and then needle biopsy, as necessary, with subsequent expectant management if all three tests were non-diagnostic. For peri- pheral lesions sputum cytology was also found to be valuable, despite the fact that it was assumed to have a sensitivity of only $20 \%$ for these lesions compared with $40 \%$ for central lesions. Life expectancy was found to be similar for sputum based and non-sputum based strategies. The least expensive strategy (needle biopsy, followed by bronchoscopy, followed by expectant manfollowed by bronchoscopy, followed by expectant management) did not include sputum cytology. However,
the incremental cost effectiveness of adding sputum cytology to this approach was $\$ 27600$ per additional life year saved, which is roughly comparable to the cost effectiveness of other widely accepted health care interventions. ${ }^{18}$

The results of this analysis varied according to the prevalence of malignant disease as demonstrated by sensitivity analysis. For central lesions sputum cytology was clearly not cost effective when the pCA was less than or equal to 0.10 , and probably not cost effective when the pCA was as high as 0.20 . Thus, bronchoscopy followed by needle biopsy followed by expectant management was the preferred strategy when the pCA was very low. For peripheral lesions the sputum first strate very low. For peripheral lesions the sputum first strategy resulted in an incremental cost effectiveness ratio of $\$ 69856$ per life year saved when the pCA was 0.30 and $\$ 463270$ per life year saved when the pCA was 0.10 when compared with a representative non-sputum based strategy. If an incremental cost effectiveness ratio of $\$ 30000-50000 /$ life year saved is adopted as a reasonable threshold, then sputum based strategies are only when the pCA for a peripheral nodule is greater than 0.40.Their decision model assumes that bronchoscopic biopsy and needle biopsy are conditionally independent, which is almost certainly incorrect, but this probably does not invalidate the conclusions reached.

Unfortunately, neither of these cost effectiveness studies included PET as a possible diagnostic test. If PET is ultimately confirmed to be highly accurate for the diagnosis of pulmonary nodules and mass lesions, the diagnosis of pulmonary nodules and mass lesions, the
cost effectiveness of sputum cytology will need to be cost effectiveness of sputum cytology will need to be
reassessed and compared with PET based strategies. In reassessed and compared with PET based strategies. In
the future the use of standard clinical and CT criteria to estimate the pCA may still be helpful in choosing the optimal strategy, although clinicians must continue to exercise caution when generalising the results of prediction rules and decision models to decision making at the individual patient level.

COST EFFECTIVENESS OF PET FOR MEDIASTINAL STAGING While much recent interest has focused on PET for SPN diagnosis, equal attention has been given to PET as a modality for mediastinal staging in non-small cell lung cancer.

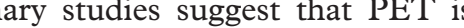
sensitive and specific for identifying malignant mediastinal lymph nodes. ${ }^{19-21} \mathrm{CT}$ scanning, in comparison, is notoriously inaccurate for mediastinal staging. 222

Gambhir and associates recently reported a cost effectiveness evaluation of PET for mediastinal staging. ${ }^{24}$ Using decision modelling this group compared costs and outcomes for PET based strategies with standard strategies that did not include PET. The standard strategy consisted of CT scanning followed by mediastinal biopsy when CT was positive, and thoracotomy when CT was negative. PET was incorporated into the diagnostic plan in one of two possible ways. In the conservative PET strategy the authors assumed that mediastinal biopsy would be performed following a positive PET, regardless of CT results, or following a 
positive result on CT if PET was negative. In the less conservative PET strategy, biopsy specimens were taken only when PET and CT results were discordant. Thus, in this strategy concordant positive results were assumed to be correct. For the base case analysis the sensitivity and specificity for CT scanning were assumed to be $67 \%$ and $73 \%$ and the corresponding values for PET were assumed to be $90 \%$ and $91 \%$.

were assumed to be $90 \%$ and $91 \%$.
The authors calculated that the conservative PET The authors calculated that the conservative PET strategy was less costly than the standard strategy, with thermore, they reported that average life expectancy increased by almost three days using the conservative minant. The less conservative PET strategy was also calculated to be less costly than the standard approach, with an estimated cost savings of $\$ 2267$ per patient. However, the less conservative PET strategy resulted in false positive staging evaluations in $1.7 \%$ of the patients. The authors did not report calculated differences in life expectancy between the less conservative PET and standard strategies, but false positive evaluations would be expected to result in missed opportunities for surgical cure and shortened survival.

Several problems appear to limit the conclusions of this analysis. Firstly, the perspective of the analysis is not clearly specified although a hospital perspective is inferred. Secondly, the time horizon of the analysis may not be sufficiently long, depending on the selected perspective. The authors limited their time frame to the period of diagnostic evaluation and initial treatment and period of diagnostic evaluation and initial treatment and
only included costs for CT scanning, PET, biopsy, and surgery. For a cost effectiveness analysis based on a societal perspective costs for subsequent care should be included, in addition to costs for diagnostic testing and initial surgical treatment. In this analysis no costs are assigned for non-surgical treatment in those thought to have unresectable disease. Thus, the $1.7 \%$ of patients have unresectable disease. Thus the $1.7 \%$ of patient ess conservative PET strategy incur no costs beyond those for CT scanning and PET. On a more technical but related note, the authors estimated costs using billed charges as a surrogate. While their cost estimates appear reasonable, charges and costs often differ widely.

Other modelling assumptions might also be challenged. These authors assumed that CT and PET results were independent. In fact, preliminary studies suggest that the test performance of PET depends partly on the results of CT scanning. More specifically, PET is most sensitive in the setting of a positive CT scan, and most specific when the CT scan is negative. ${ }^{25}$ This potentially limits the usefulness of PET since ideally PET should be very specific following a positive CT scan if one wishes to minimise the wishes to minic following a positive CT scan if one gical cure. Gambhir and colleagues also limit the range of possible strategic alternatives. For selected patients with non-small cell lung cancer at very low risk for mediastinal lymph node involvement it is conceivable that prompt surgery without diagnostic testing would be the most cost effective strategy, but this option is not considered. Finally, the authors assu is not considered. sensitivity of mediastinoscopy and other biopsy procedures is known to be less than perfect. ${ }^{26}$

\section{LEARNING POINTS}

* The solitary pulmonary nodule (SPN), usually an asymptomatic incidental radiological finding, is one of the more common methods of presentation of bronchogenic carcinoma, currently the leading cause of death from cancer in both men and women.

* If diagnosed and treated promptly, the malignant SPN will have a five year survival rate of $40-80 \%$; the smaller the nodule at the time of resection, the higher the five year survival rate.

* It is possible to calculate the probability that the nodule is malignant (pCA) by Bayesian means from clinical and radiological findings, thereby providing assistance in the choice of definitive investigative/therapeutic strategies.

* Standard strategies include immediate thoracotomy, non-thoracotomy biopsy of the nodule (usually by transthoracic needle aspiration), and "watch and wait" with serial chest radiographs.

* Growth of the nodule during the watch and wait period is an indication for prompt thoracotomy.

* In a given patient the calculated value for pCA indicates which strategy is likely to be most effective in terms of life expectancy.

* Positron emission tomography (PET) is a new imaging process which appears to have a high sensitivity and specificity in detecting malignancy in the SPN, the regional and mediastinal lymph nodes, and in distant organs but further studies, particularly cost effectiveness analyses, are needed to determine its exact role.

* Despite criticisms and controversy over the value of sputum analyses, a recent study suggests that it is cost effective in certain circumstances, depending upon the pCA and whether the nodule has a central or peripheral location. 


\section{Conclusions}

It is often useful to estimate the probability of malignancy in SPNs in order to guide management decisions. Although formal prediction guidelines have been developed for this purpose, their applicability to the particular clinical setting must be considered. Preliminary reports suggest that PET may be an accurate and useful non-invasive imaging test for detecting malignant SPNs. Results from an ongoing Veterans Administration Cooperative study will better define the accuracy and cost effectiveness of PET for SPN diagnosis and tumour staging. PET has the great advantage that a single noninvasive test can simultaneously provide information about the primary lung nodule, the mediastinal nodes, and possible distant metastases to other organs.

For the diagnosis of lung cancer sputum cytology is often highly cost effective, especially for central lung lesions with a high pre-test probability of malignancy, and it also appears to have a legitimate but reduced role in the investigation of patients with peripheral lung nodules. The test is simple, inexpensive, non-invasive, and rapidly reported, and we would disagree with the conclusions of Goldberg-Kahn et $a l^{16}$ in the second introductory article. The policy of assessing pCA as a guide to management choices is still widely accepted. Expectant management with radiological monitoring (watchful waiting) as the initial strategy appears indicated if the pCA is low, and thoracotomy as the initial strategy is advisable if the pCA is high. High resolution CT scanning will occasionally provide definitive identification of benignity in SPN without requiring needle biopsy, and PET may possibly obviate this need in many instances.

Given current concerns about rising health care expenditures, cost effectiveness studies are likely to play an increasingly important role in the evaluation of new and existing technologies for the diagnosis of lung cancer and the investigation of SPNs. The challenges will include defining the most cost effective diagnostic tests and strategies, and determining under what cirand strategies, and determining under what cir-
cumstances and in which populations these approaches cumstances and in which populations these approaches
might best be employed. The ultimate goal of such might best be employed. The ultimate goal of such
evaluations is to identify interventions that produce the greatest possible health benefits at costs that are judged to be reasonable and worthwhile.

\footnotetext{
1 Lillington GA. Management of solitary pulmonary nodules. Disease-a-
Month 1991;37:271-318.
}

2 Sox HC Jr, Blatt MA, Higgins MC, et al. Medical decision making. 3 Kunstaetter R, Workove N, Kreisman $\mathrm{H}$, et al The solitary pulmonary nodule: decision analysis. Med Decis Making 1985,5:61-75. Cummings SR, Lillington GA, Richard R. Estimating the probability
of malignancy in solitary pulmonary nodules: a Bayesian approach. $D$ solitary pulmonary

5 Gurney J, Lyddon DM, McKay JA. Determining the likelihood of malignancy in solitary pulmonary (1) Dewan NA, Shehan CJ, Reeb SD, et al. Likelihood of malignancy in a solitary pulmonary nodule. comparison of Bayesian analysis and results of FDG-PET scan. Chest 1997;112:416-22.

Duhaylongsod FG, Lowe VJ, Patz EF Jr, et al. Detection of primary and recurrent lung cancer by means of F-18 fluorodeoxyglucose
positron emission tomography (FDG PET). $\mathscr{f}$ Thorac Cardiovasc Surg 1995;110:130-9.

Bury , Dowlati A, Paulus P, et al. Evaluation of the solitary pulmonary
nodule by positron emission tomography imaging. Eur Respir f 1996; 9:410-4.

Gupta NC, Maloof J, Gunel E. Probability of malignancy in solitary
pulmonary nodules using fluorine-18-FDG and PET. $尹$ Nucl Med pulmonary nodules using fluorine-18-FDG and PET. F Nucl Med
1996;37:943-8.

11 Cummings SR, Lillington GA, Richard R. Managing solitary pulmonary 1986;134:453-60

12 Barlow PW, Beck JR: The solitary pulmonary nodule: a decision analysis. Chest 1985;88:45S
Russell L Siegel Je

guide to resource allocati $\mathrm{N}$, et al. Cost-effectiveness analysis as a $\mathrm{M}$, Siegel J, Russell L, et al, eds. Cost-effectiveness in health and medicine. New Mork. Oxford Universty Cress, 1996: 3-24.

In: Gold M, Siegel I, Russell L, et al. Cost-effectiveness in health as. medicine. New York: Oxford University Press, 1996: 82-134. of chest CT Goldberg-Kahn B, Healy JC, Bishop JW. The cost of diagnosis: a
comparison of four different strategies in the workup of solitary comparison of four different strategies in the workup or solitary
radiographic lung lesions. Chest 1997;111:870-6. 17 Raab S, Hornberger J Raffin T The importance of

17 Raab S, Hornberger J, Raffin T. The importance of sputum cytology
in the diagnosis of of lung cancer. Chest 1997;112:937-45. O'Brien B, Heyland D, Richardson W, et al. User's guides to the medical literature. XIII. How to use an article on economic analysis of clinical my patients? FAMA 1997;277:1802-6. Wahl R, Quint L, Greenough R, et al. Staging of mediastinal non-small prospective evaluation. Radiology 1994;191:371-7.
Chin R, Ward R lung cancer with positron emission tomography. Am $\mathcal{Y}$ Respir Crit Care 21 Valk PE, Pounds TR, Hopkins DM, et al. Staging non-small cell lung cancer by whole body positron emission tomographic imaging. An Thorac Surg 1995;60:1573-82.
Webb W, Gatsonis C, Zerhouni E, et al. CT and MR imaging in staging non-small cell bronchogenic carcinoma: report of the radiologic diagnostic oncology group. Radiology 1991;178:705-13.

23 McLoud T, Bourguin P, Greenberg R, et al. Bronchogenic carcinoma: node mapping and sampling. Radiology 1992;182:319-23. 4 Gambhir SS, Hoh CK, Phelps ME, et al. Decision tree sensitivity analysis for cost-effectiveness of FDG-PET in the staging and management of Gould MK, Owens DK. Positron emission tomography for mediastinal staging in non-small cell lung cancer: a meta-analysis. Am f Respi Luke W, Pearson F, Todd T, et al. Prospective evaluation of media-
stinoscopy for assessment of carcinoma of the lung. $₹$ Thorac Cardiovasc 